This is an electronic reprint of the original article. This reprint may differ from the original in pagination and typographic detail.

Author(s): Nauha, Elisa; Ojala, Antti; Nissinen, Maija; Saxell, Heidi

Title: $\quad$ Comparison of the polymorphs and solvates of two analogous fungicides-a case study of the applicability of a supramolecular synthon approach in crystal engineering

Year: $\quad 2011$

Version:

Please cite the original version:

Nauha, E., Ojala, A., Nissinen, M., \& Saxell, H. (2011). Comparison of the polymorphs and solvates of two analogous fungicides - a case study of the applicability of a supramolecular synthon approach in crystal engineering. CrystEngComm, 13(15), 4956-4964. https://doi.org/10.1039/C1CE05077J

All material supplied via JYX is protected by copyright and other intellectual property rights, and duplication or sale of all or part of any of the repository collections is not permitted, except that material may be duplicated by you for your research use or educational purposes in electronic or print form. You must obtain permission for any other use. Electronic or print copies may not be offered, whether for sale or otherwise to anyone who is not an authorised user. 


\title{
Comparison of the polymorphs and solvates of two analogous fungicides - a case study of the applicability of a supramolecular synthon approach in crystal engineering
}

\author{
Elisa Nauha, ${ }^{a}$ Antti Ojala ${ }^{b}$, Maija Nissinen ${ }^{a}{ }^{a}$ and Heidi Saxell ${ }^{b}$

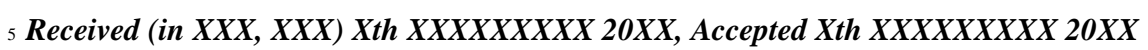 \\ DOI: 10.1039/b000000x
}

The polymorphism and solvate formation of thiophanate-ethyl (TE), a fungicidal active, was investigated by solvent crystallization and compared to a close analogue, thiophanate-methyl (TM). Four polymorphs and seven solvates of TE were found and structurally compared with the previously found two

${ }_{10}$ polymorphs and fourteen solvates of TM by analyzing the hydrogen bonding patterns and using fingerprint plots, packing coefficients and lattice energies. TE and TM have the same functional groups that can build identical supramolecular synthons. Despite the strong similarities, the polymorphs and solvates of the two actives show significant differences in hydrogen bonding and packing. The results demonstrate the challenges in using a supramolecular synthon approach, and promote the importance in

${ }_{15}$ finding methods to also make use of packing effects and lipophilic interactions in crystal engineering.

In a crystallization process, the molecules arrange in an energetically favorable way. The energetic incentive of favorable intermolecular interactions, the strongest of which are hydrogen bonds for neutral molecules, and reduction of void space, 20 negotiate for the best arrangement of molecules in the crystal. For most of organic molecules, there are several ways to arrange, causing polymorphs to appear. ${ }^{1}$ In a solvent based polymorph screening, it is typical to also find solvates, that are often more stable than the polymorphs of a compound in the solvate forming ${ }_{25}$ solvent. $^{2}$ The crystallization outcome then depends on the kinetics of crystallization and the presence of other contributing additives, either the solvent or other species, in the crystallization medium.

The aim in modern crystal engineering is the targetted 30 discovery of multicomponent crystals, i.e. cocrystals. As solvates can be considered to belong to a subgroup of cocrystals, the study of solvate formation can give an insight for designing cocrystals. Suitable cocrystals can enable the tuning of the crucial physical and chemical parameters such as the solubility, vapour pressure, 35 crystal habit etc., of a an active ingredient in pharmaceutical, agrochemical or other areas. ${ }^{3}$ The prevailing model for cocrystal engineering is using a supramolecular synthon approach, where supramolecular synthons, which consist of intermolecular interactions (especially hydrogen bonds), are viewed as bonds for 40 the construction of supermolecules, i.e. crystals. ${ }^{4}$

Crystal structures give the most definite understanding of the crystal packing and intramolecular interactions, and help give strategies for crystal engineering. The conformation of the molecule, hydrogen bonding and other intermolecular 45 interactions as well as the packing can be assessed with the help of a number of tools. The packing coefficients ${ }^{5}$, fingerprint plots ${ }^{6}$ and lattice energies ${ }^{7}$ can be calculated to ensure not missing vital features when assessing the structure visually. The use of graph set symbols ${ }^{8}$ for hydrogen bonding facilitate the easy comparison 50 of hydrogen bonding in similar structures.

We have previously investigated thoroughly the polymorphism and solvate formation ${ }^{9}$, as well as cocrystal formation ${ }^{10}$, of the fungicidal active thiophanate-methyl (TM, dimethyl 4,4'-(ophenylene)bis(3-thioallophanate), Scheme 1. According to our 55 findings, TM has two conformational polymorphs and at least fourteen solvates. TM is an excellent example of a small molecule with possibilities to serve as a versatile supramolecular building block, that has conformational flexibility and is able to form host-guest frameworks. To get an understanding of the 60 applicability of a supramolecular synthon approach, we investigated the polymorphism of a close analogue of TM that differs only with two $\mathrm{CH}_{2}$ groups. Thiophanate-ethyl (TE, diethyl 4,4'-(o-phenylene)bis(3-thioallophanate), Scheme 1) is a fungicide that has been withdrawn from the European market ${ }^{11}$ ${ }_{65}$ because of safety issues and the availability of an efficient alternative, mainly TM, which is identical in fungicidal function and from a supramolecular synthon perspective. This paper reports the four found polymorphs of TE, as well as the analysis of the achieved single crystal structures of three polymorphs and 70 seven solvates, as comparison with the previously reported TM structures. 
TM

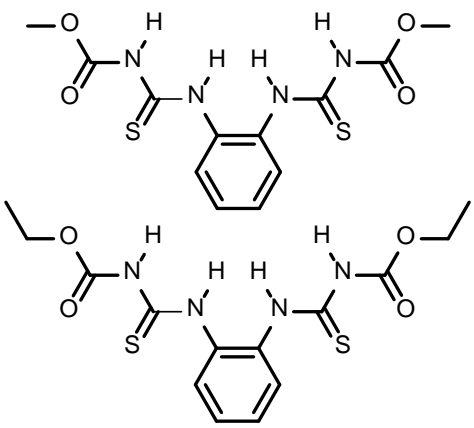

Scheme 1 Molecular structures of thiophanate-methyl (TM) and thiophanate-ethyl (TE)

\section{Experimental}

\section{${ }_{5}$ Materials}

TE of $99 \%$ purity from Chem Service, distilled water and solvents of analytical purity (min 99\%) were used in the crystallization experiments. To save material TE was reused without purification.

\section{${ }_{10}$ Crystallizations}

Approximately $20 \mathrm{mg}$ of TE was dissolved in $5 \mathrm{ml}$ of solvent (acetonitrile, 1:1 acetonitrile:water, acetone, ethanol, methanol, DCM, chloroform, pyridine, dioxane and toluene:methanol). The solution was transferred to a clean test tube, which was covered 15 with parafilm with a few holes. The solvent was left to evaporate in room temperature.

\section{PXRD}

For powder X-ray diffraction analysis the original TE was hand grinded, pressed to a zero background silicon plate and measured 20 on a PANalytical X'Pert Pro system in reflection mode with $\mathrm{CuK} \alpha 1$-radiation. A $2 \theta$-angle range of $5-40^{\circ}$ and a step time of $60 \mathrm{~s}$ were used with step resolution of $0.0167^{\circ}$. Figures were drawn with $\mathrm{X}^{\text {'Pert HighScore Plus }}{ }^{12}$.

\section{Single crystal X-ray diffraction}

25 The X-ray diffraction data was collected on Nonius Kappa CCDdiffractometer with Apex II detector at $173 \mathrm{~K}$, using graphitemonochromated $\mathrm{CuK} \alpha$ radiation $(\lambda=1.54178 \AA)$, or in the case of the pyridine solvate graphite-monochromated MoK $\alpha$ radiation $(\lambda=0.71073 \AA)$. Absorption correction was performed with 30 Denzo-SMN $1997^{13}$. The structures were solved using direct methods, refined, and expanded by using Fourier techniques with the SHELX-97 software package ${ }^{14}$. All non-hydrogen atoms, except for the disordered toluene molecule, were refined anisotropically. Hydrogen atoms were placed in idealized 35 positions or found from the electron density map (hydrogen bonding $\mathrm{N}-\mathrm{H}$ hydrogens), and included in structure factor calculations. The $\mathrm{N}-\mathrm{H}$ hydrogen atoms found in the electron density map were restrained to a distance of $0.91 \AA$ to give the best fit to the X-ray data and to ensure stable refinement. Pictures 40 of the structures were drawn with Mercury. ${ }^{15}$ Crystal data and collection parameters are presented in Table 1 and Table 3.

In the dioxane I, DCM and chloroform solvates the solvent molecules are disordered over a symmetry axis in two positions. There are voids of $42 \AA^{3}$ in the toluene solvate structure that are
${ }_{45}$ surrounded by the ethyl groups of TE and the toluene molecules making the inclusion of water unlikely due to hydrophobic surroundings. The toluene molecule is on a general position, but disordered over two positions that are coplanar and in a $180^{\circ}$ angle relative to each other. In Form III there are voids of around ${ }_{50} 45 \AA^{3}$ between the arms of one of the symmetry unequivalent molecules with no significant residual electron density.

\section{Lattice energy calculations}

The lattice energies $\left(E_{\text {latt }}\right)$ of the polymorphs of TE and TM were calculated using the Cerius ${ }^{2}$ program package. ${ }^{16}$ All computations

55 were performed with the default settings of the program and following the previously published procedure. ${ }^{7}$ Atomic charges were assigned by QEq method and cross checked with AM1 which yielded essentially similar results. The $E_{\text {latt }}$ values were calculated with a Dreiding II force field for the energetically 60 optimized structures. During structure optimization the molecules were treated as rigid objects and only the unit cell dimensions were allowed to change. The optimized unit cells diverged less than $3 \%$ of the original values.

The approximated modeling yielded generally similar 65 energetic values for the polymorphs in a meaningfull energy window. However, due to the inaccurate nature of the method (e.g. lack of polarizability), the absolute values are not reliable and only the relative energies should be judged. Precise $E_{\text {latt }}$ calculations would need expansive DFT calculations and/or 70 extensive calibration of the force fields to reach the accuracy that is needed to judge the total energies which often diverge 1-2 $\mathrm{kcal} / \mathrm{mol}$ between the polymorphs. ${ }^{17}$

Table 1 Crystal data and collection parameters of the polymorphs of TE

\begin{tabular}{llll}
\hline & TE-form I & TE-form II & TE-form III \\
\hline Chemical formula & $\mathrm{C}_{14} \mathrm{H}_{18} \mathrm{~N}_{4} \mathrm{O}_{4} \mathrm{~S}_{2}$ & $\mathrm{C}_{14} \mathrm{H}_{18} \mathrm{~N}_{4} \mathrm{O}_{4} \mathrm{~S}_{2}$ & $\mathrm{C}_{14} \mathrm{H}_{18} \mathrm{~N}_{4} \mathrm{O}_{4} \mathrm{~S}_{2}$ \\
Formula Mass & 370.44 & 370.44 & 370.44 \\
Crystal system & Triclinic & Monoclinic & Triclinic \\
$a / \AA$ & $7.9911(1)$ & $4.7271(1)$ & $10.7333(5)$ \\
$b / \AA$ & $9.6750(2)$ & $16.0239(3)$ & $11.8079(7)$ \\
$c / \AA$ & $12.5109(3)$ & $22.6450(4)$ & $16.2174(12)$ \\
$\alpha /{ }^{\circ}$ & $69.055(3)$ & 90.00 & $95.293(3)$ \\
$\beta /{ }^{\circ}$ & $81.270(3)$ & $93.750(3)$ & $100.405(4)$ \\
$\gamma /{ }^{\circ}$ & $73.033(3)$ & 90.00 & $113.044(4)$ \\
$\mathrm{V} / \AA^{3}$ & $862.81(3)$ & $1711.61(6)$ & $1829.9(2)$ \\
Temperature/K & $173(2)$ & $173(2)$ & $173(2)$ \\
Space group & $P-1$ & $P 2(1) / c$ & $P-1$ \\
$Z$ & 2 & 4 & 4 \\
Meas. reflns. & 4039 & 4691 & 8675 \\
Indep. reflns. & 2936 & 2931 & 6132 \\
$R_{\text {int }}$ & 0.0848 & 0.0616 & 0.1113 \\
$R_{1}(I>2 \sigma(I))$ & 0.0458 & 0.0550 & 0.0603 \\
$w R\left(F^{2}\right)(I>2 \sigma(I))$ & 0.1235 & 0.1120 & 0.1413 \\
$R_{1}($ all data) & 0.0520 & 0.0898 & 0.0963 \\
$w R\left(F^{2}\right)($ all data) & 0.1299 & 0.1283 & 0.1633 \\
$\mathrm{GOF}$ on $F^{2}$ & 1.031 & 1.060 & 1.026 \\
& & & \\
& & &
\end{tabular}

\section{${ }_{75}$ Results and discussion}

Four polymorphs and seven solvates of thiophanate-ethyl were found during the solvent screening experiments. The polymorphs were named in order of discovery as forms I-IV. Samples crystallized from acetonitrile and methanol produced crystals of 

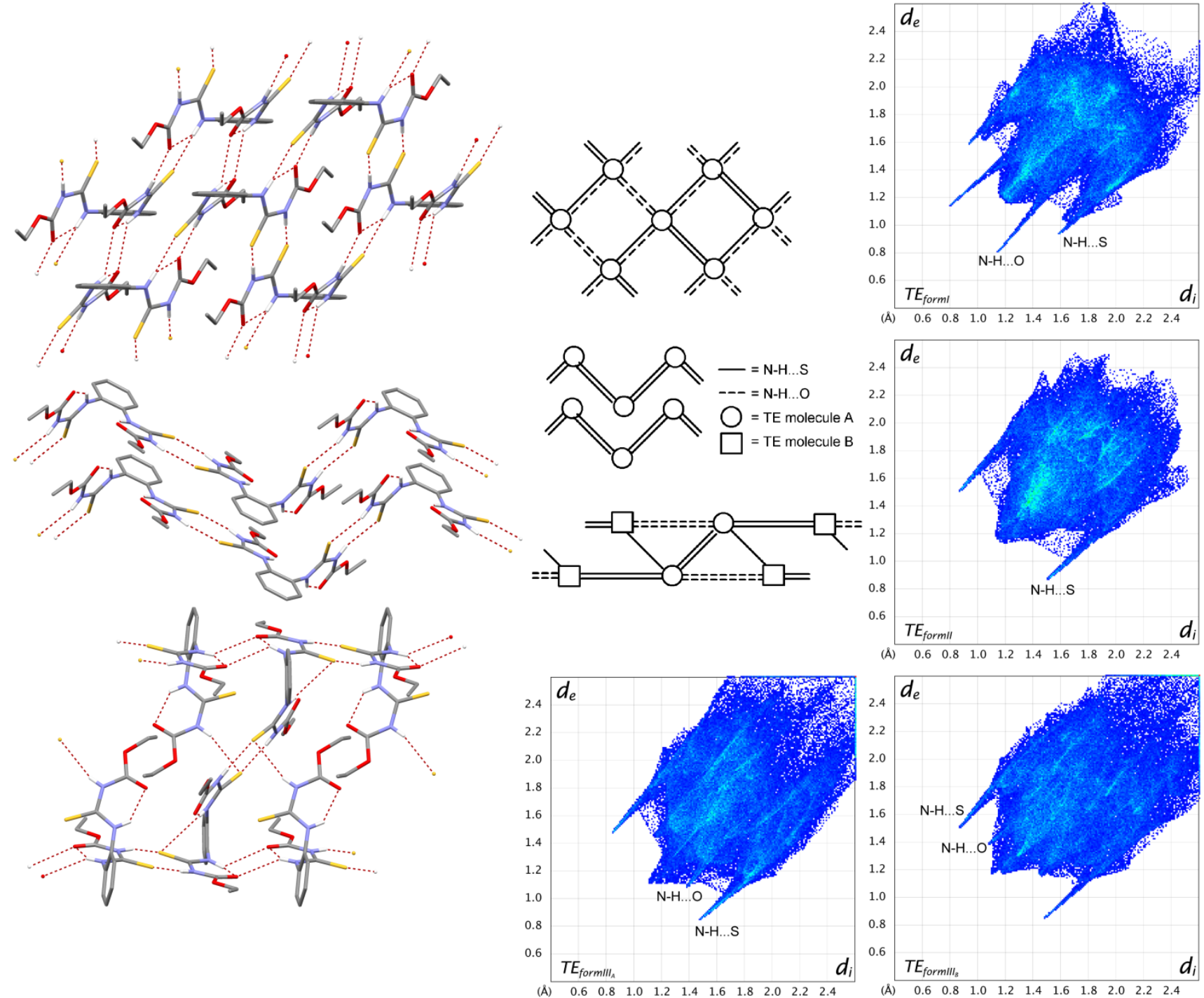

Fig. 1 Hydrogen bonding in the sheets of TE form I (top), in the chains of TE form II (middle) and the double chains of TE form III (bottom) with nonhydrogen bonding hydrogen atoms removed for clarity The simplified diagrams of the hydrogen bonding and the fingerprint plots of forms I-III.

TE form I and a sample crystallized from ethanol produced 5 crystals of form II. Samples crystallized from 1:1 acetonitrile:water concomitantly produced crystals of form I and form II. Form III was crystallized from a methanol solution that also contained sodium acetate. The pyridine, toluene, acetone, dioxane, DCM and chloroform samples produced solvate 10 crystals. Two polymorphic solvates (dioxane I and II) were crystallized from dioxane in the course of trying to get better crystals of the first one. The fourth polymorph of TE was identified by powder diffraction, but no single crystal structure was obtained. Form IV appears upon desolvation of the 15 isomorphous solvates (acetone, dioxane I, DCM and chloroform).

\section{TE Form I}

In TE form $\mathrm{I}$ as in all the structures of $\mathrm{TE}$ there is an intramolecular $\mathrm{N}-\mathrm{H} \cdots \mathrm{O}=\mathrm{C}$ hydrogen bond in a $\mathrm{S}(6)$ motif in both the arms of the molecule that restricts the conformation of the 20 arms. The molecules of TE in form I are connected to each other by three kinds of hydrogen bonding motifs, of which one consists of two N-H••s hydrogen bonds R2,2(8), another of two N-
$\mathrm{H} \cdots \mathrm{O}=\mathrm{C}$ hydrogen bonds $\mathrm{R} 2,2(12)$ and the third of one $\mathrm{N}-\mathrm{H} \cdots \mathrm{S}$ hydrogen bond and one $\mathrm{N}-\mathrm{H} \cdot \bullet \mathrm{O}=\mathrm{C}$ hydrogen bond $\mathrm{R} 2,2(10)$ 25 (Fig. 1). The TE molecules thus connect into two-dimensional sheets that stack up on each other. The benzene and ethyl groups of TE protrude to both sides of the sheets, and there are face-toface $\pi-\pi$ stacking interactions, with ring distances of approximately $3.5 \AA$, between the sheets.

\section{TE Form II}

The TE molecules in form II build up hydrogen bonded chains with the same R2,2(8) motif consisting of two $\mathrm{N}-\mathrm{H} \bullet \bullet$ S hydrogen bonds as in form I (Fig. 1). Unlike in form I there are only intramolecular $\mathrm{N}-\mathrm{H} \cdots \mathrm{O}=\mathrm{C}$ hydrogen bonds. The chains arrange 35 parallel to each other guided by $\pi-\pi$ stacking interactions between the benzene rings and dipole-dipole interactions between the carbonyls in adjacent chains. The parallel chains make up sheetlike structures that stack up on each other in a manner similar to that in form I with benzene and ethyl groups of TE protruding to 40 both sides of the sheets. 


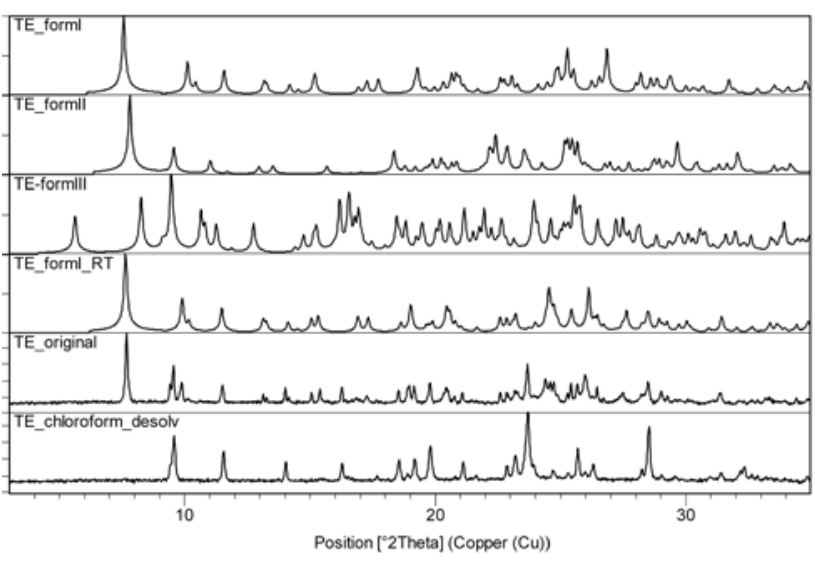

Fig. 2 From top to bottom calculated PXRD patterns of TE form I-III at $173 \mathrm{~K}$ and form I at RT, the PXRD pattern of the original TE sample and of form IV from desolvation of the chloroform solvate.

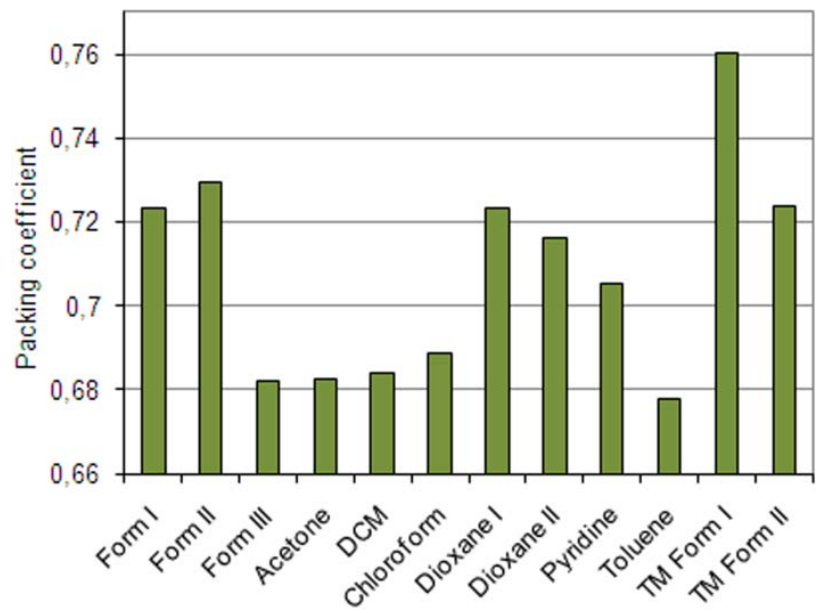

Fig. 3 Packing coefficients of the single crystal structures of TE and the polymorphs of TM

\section{TE Form III}

Unlike in forms I and II, the Z' of form III is 2. The molecules 10 build hydrogen bonded chains of the two symmetrically unequivalent molecules (A and B) connected with two $\mathrm{N}-\mathrm{H} \cdots \mathrm{S}$ hydrogen bonds in the binary level R2,2(8) arrangement and with two $\mathrm{N}-\mathrm{H} \cdots \mathrm{O}$ hydrogen bonds in a binary level R2,2(12) arrangement (Fig. 1). This chain involves only one arm of the 15 molecules. Two chains are connected to each other via $\mathrm{N}-\mathrm{H} \cdots \mathrm{S}$ hydrogen bonds on the other arms of the molecules with a $\mathrm{R} 2,2(8)$ arrangement and a D1,1(2) motif. There are also intramolecular $\mathrm{N}-\mathrm{H} \cdots \mathrm{S}$ hydrogen bonds in a S(7) motif.

Solution NMR evidence suggest that there is some interaction 20 between the $\mathrm{N}-\mathrm{H}$ hydrogens and the acetate present in the crystallization, which could have influenced the aggregation of TE molecules initiating the crystallization of this form. The Z' of 2 and the presence of small voids suggests that this could be a case of crystallization "on the way". ${ }^{18}$

\section{${ }_{25}$ TE Form IV}

Form IV was found to be a desolvation product of the isomorphous solvates and can be identified by the PXRD pattern. The original sample from ChemService was found to be a combination of form I and form IV (PXRD in Fig. 2). This can be 30 most clearly seen in the peaks caused by form IV at 9.5, 16.3 and

\section{$23.7^{\circ} 2 \theta$.}

The unit cell of this polymorph was determined from the powder pattern using X'Pert HighScore Plus and the most likely candidate is a monoclinic cell with a possible spacegroup of $\mathrm{C} 2 / \mathrm{c}$ 35 with $a=11.50, b=18.76, c=9.22, \beta=112.24^{\circ}$ and $V=1846.5 \AA^{3}$. A structure in this cell can easily be imagined from the isomorphous solvate structures by removing the solvent and moving the TE molecules to fill the formed voids. Efforts to solve the structure from powder data are currently underway.

\section{${ }_{40}$ Packing efficiency of the polymorphs}

The fingerprint plots in Fig. 1 show a graphical representation of the packing in forms I to III. The packing coefficients (Fig. 3) of the structures were also determined ${ }^{19}$ in order to get a numerical estimate of the packing efficiency. According to the results, form 45 II packs the most tightly with form I in second place. The packing coefficient and the hydrogen bonding seem to explain the similar stability of forms I and II since form I has more and stronger intermolecular hydrogen bonds than form II, but form II packs more tightly. Form III, on the other hand, packs very loosely and

50 is expected to be less stable even though there is a lot of hydrogen bonding.

The packing coefficients were calculated also for the previously reported polymorphs of TM. TM form I is clearly more densely packed than form II. This can also be seen in the 55 fingerprint plots (Fig 4). From the fingerprint plots one can also say that the $\mathrm{N}-\mathrm{H} \cdots \mathrm{S}$ hydrogen bonds in form II are shorter, indicating a stronger nature. The stronger $\mathrm{N}-\mathrm{H} \cdots \mathrm{O}$ hydrogen bonds are, however, somewhat shorter for form I.

Table 2 Lattice energies of the polymorphs of TE and TM in kcal/mol

\begin{tabular}{lcccc}
\hline Polymorph & $\mathbf{E}_{\mathbf{v d w}}$ & $\mathbf{E}_{\mathbf{C}}$ & $\mathbf{E}_{\mathbf{H}}$ & $\mathbf{E}_{\text {latt }}$ \\
\hline TE Form I & $-33,96$ & $-1,89$ & $-6,08$ & $-41,94$ \\
TE Form II & $-38,38$ & 4,09 & $-6,02$ & $-40,31$ \\
TE Form III & $-31,81$ & $-3,90$ & $-3,41$ & $-39,11$ \\
TM Form I & $-33,99$ & $-8,54$ & $-3,18$ & $-45,71$ \\
TM Form II & $-29,79$ & $-1,20$ & $-6,13$ & $-37,11$ \\
& & & & \\
\hline
\end{tabular}

\section{Experimental observations on the stability}

Thermal methods to determine the stabilities of the forms could not be used because TE decomposes when melting or heated for prolonged periods. Very few experiments were done to find out 65 the stability order of the polymorphs, because the forms could not be reproduced in large quantities. The occasional concomitant crystallization suggests that form I and form II are quite similar in stability, but attempts to crystallize form II in larger amounts resulted only in form I. The kinetics of the crystallizations are 70 likely the cause of this behavior. When the original sample, a mixture of form I and form IV, was slurried in MeCN-water mixtures, it changed to pure form I.

\section{Lattice energies of the polymorphs}

The lattice energies $\left(E_{\text {latt }}\right)$ of the polymorphs (Table 2) were 75 calculated to get understanding of the energies governing the packing of the forms.

The computed $\mathrm{E}_{\text {latt }}$ of different $\mathrm{TE}$ polymorphs are inside 3 $\mathrm{kcal} / \mathrm{mol}$ and indicate a stability order of I > II > III which is supported by the available experimental observations. The Van 
der Waals energy $\left(E_{v d w}\right)$ component, which describes the nonbonding attractive and repulsive interactions, is typically most accurately modeled by the force field calculations. This is considerably larger, indicating denser packing, for form II 5 compared to forms I and III, which is in-line with the crystallographical densities of the polymorphs. Also the fingerprint plots (Fig. 1) show average shorter intermolecular distances for form II. The calculated coulombic energies $\left(E_{C}\right)$, which describe the electrostatic interactions in the crystal, show a 10 discrepancy in that the energy value for TE form II is positive, indicating an unfavorable interaction. The cause for this is likely the short intra- and intermolecular $\mathrm{C}=\mathrm{O} \cdots \mathrm{O}=\mathrm{C}$ distances which are in the range of 3.05 to $3.11 \AA$. The $\mathrm{E}_{\mathrm{c}}$ calculations, however, are problematic due to inaccurate determination of the electronic 15 charges and should be judged with some caution. ${ }^{20}$ Calculated hydrogen bond energies $\left(E_{H}\right)^{21}$ gave similar energies for forms I and II, but clearly lower for form III. The result is somewhat surprising as the fingerprint plots indicate that form I has some substantially stronger intermolecular hydrogen bonds than the 20 two other polymorphs. However, the high $\mathrm{E}_{\mathrm{H}}$ term of form II can be explained by the intramolecular $\mathrm{N}-\mathrm{H} \cdots \mathrm{O}=\mathrm{C}$ bonds which are most linear and shortest in form II.

The energy calculations of TM polymorphs gave significantly higher $\mathrm{E}_{\mathrm{vdw}}$ for form I as also indicated by the packing coefficient 25 and the fingerprint plot Fig. 4, but also indicate stronger total hydrogen bonding $\left(E_{H}\right)$ in the crystals of form II. In any case, the total $E_{\text {latt }}$ shows that form $I$ is clearly more stable of the two polymorphs, though, the relatively high $\mathrm{E}_{\mathrm{C}}$ of form I might be overestimated and thus exaggerate the total energy difference.

\section{${ }_{30}$ Structural comparison to TM}

A hydrogen bonding diagram and fingerprint plots of TM forms I and II were drawn for comparison (Fig 4). The polymorphs of TE and TM do not have matching hydrogen bonding arrangements. TE form I and TM form II are very similar on a quick glance but 35 the hydrogen bonds are different. The structure of the hydrogen bonded sheets in TE form I is, however, identical to those of TM in the 1,2-dichlorobenzene solvate ${ }^{9}$ (fingerprint plot in Fig.4). In the TM solvate the solvent molecules are located between the sheets that are consequently further away from each other than in 40 the TE polymorph. The extra methyl groups of TE possibly enables closer packing of the sheets without the need for guests. A conformation where the sulfur atoms are on the same side of the benzene ring is interestingly only seen in these two structures of all the 26 solved structures for $\mathrm{TM}^{9}$ and $\mathrm{TE}$.

45 The conformation of the TE molecule in form II is most like that of the TM molecule in the benzene solvate ${ }^{9}$. The other arm of the molecule, however, is not as planar as in TE form II and consequently the intramolecular $\mathrm{C}=\mathrm{O} \cdots \mathrm{O}=\mathrm{C}$ distance that in TE form II is $3.11 \AA$ is now $3.66 \AA$. The hydrogen bonding pattern is 50 somewhat different in these two structures with the TM solvate having chains of TM connected with the common R2,2(8) motif as well as a R2,2(8) ether $\mathrm{O}$ pairing motif.

The hydrogen bonded chains of TE and packing in form III is most similar to that in the very stable ethanol and methanol 55 solvates of TM, which also have a Z' of 2. The D1,1(2) motif and the unused $\mathrm{C}=\mathrm{S}$ acceptor in one of the TE molecules in form III is replaced in the TM solvents with hydrogen bonds to the solvent molecules.
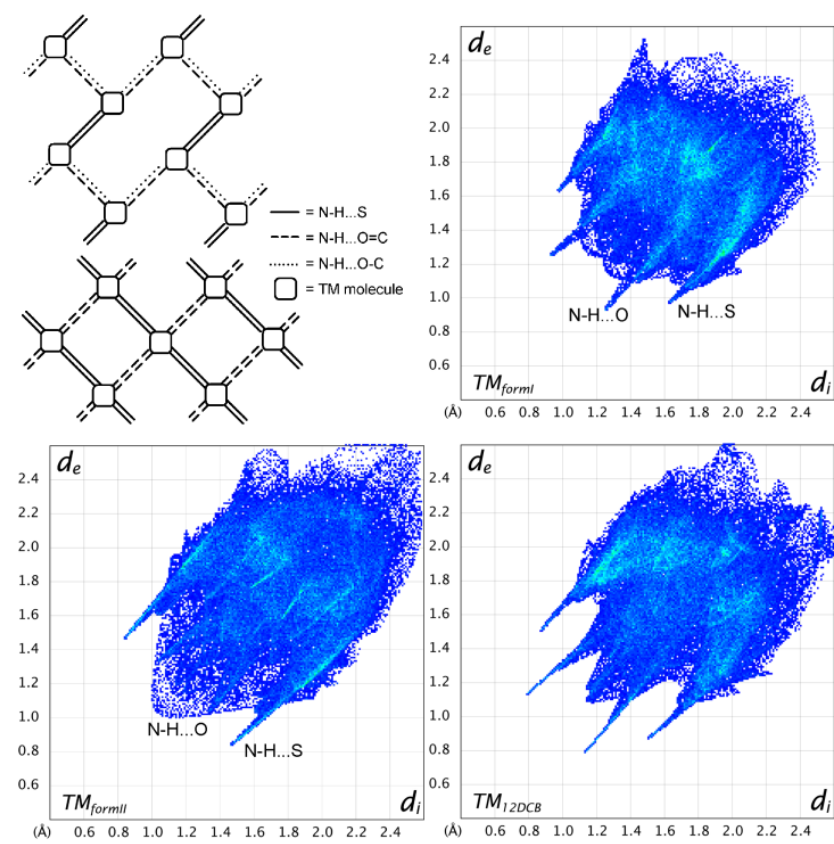

60 Fig. 4 Hydrogen bonding diagram and fingerprint plots of TM form I, form II and the 1,2-dichlorobenzene solvate.

The same hydrogen bonding motifs are consistently found in

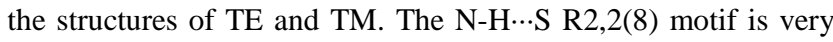
prevalent in forming chains as well as pairs of molecules. The ${ }_{65} \mathrm{R} 2,2(12) \mathrm{C}=\mathrm{O} \cdots \mathrm{H}-\mathrm{N}$ motif, that is seen for TE only in form I and form III, is much more common for structures of TM where 12 out of 16 structures have this motif. Mixed pairing of hydrogen bonds to sulfur and oxygen are shown in only a couple of structures like the R2,2(10) motif in TE form I. Some forms of ${ }_{70} \mathrm{TM}$ also use the ether $\mathrm{O}$ for hydrogen bonding, but this is not seen in the structures of TE.

\section{TE solvates}

Structures of four isomorphous solvates (acetone, dioxane I, DCM and chloroform), a toluene solvate, a dioxane solvate 75 polymorph and a pyridine solvate of TE were acquired.

In all the solvates the hydrogen bonding consists of $\mathrm{N}-\mathrm{H} \cdots \mathrm{S}$ hydrogen bonds in the R2,2(8) motif with no intermolecular $\mathrm{N}$ $\mathrm{H} \cdots \mathrm{O}=\mathrm{C}$ bonds. In the isomorphous solvates the R2,2(8) motifs connect into chains (Fig. 5a) that pack parallel to each other with $80 \pi$ - $\pi$ stacking interactions to neighboring parallel chains with ring distances of approximately $3.4 \AA$. The solvent molecules are located between the arms of the TE molecules in channels running through the structure in the direction of the crystallographic c-axis (Fig. 5b).

85 In the pyridine and dioxane II solvates the $\mathrm{N}-\mathrm{H} \cdots \mathrm{N}$ or $\mathrm{N}-\mathrm{H} \cdots \mathrm{O}$ $\mathrm{D}(2)$ motifs to the solvent, respectively, block the formation of chains of TE molecules leaving only hydrogen bonded pairs of TE molecules (Fig. 6a-b) connected with the R2,2(8) motif.

In the toluene solvate the R2,2(8) motifs connect into spiraling 90 chains, causing the chirality of the structure (Fig. 7). The parallel spirals intertwine making up stacks of benzene rings running through the structure. The disordered toluene molecules are located in channels running in the same direction as the elongation of the spirals. 
Table 3 Crystal data and collection parameters of the solvates of TE

\begin{tabular}{|c|c|c|c|c|c|c|c|}
\hline & TE-acetone & TE-DCM & TE-chloroform & TE-dioxane $\mathrm{I}^{\mathrm{a}}$ & TE-dioxane II & TE-pyridine & TE-toluene $^{\mathrm{b}}$ \\
\hline Chemical formula & $\begin{array}{l}\mathrm{C}_{14} \mathrm{H}_{18} \mathrm{~N}_{4} \mathrm{O}_{4} \mathrm{~S}_{2} \\
\text { - } \mathrm{C}_{3} \mathrm{H}_{6} \mathrm{O}\end{array}$ & $\begin{array}{l}\mathrm{C}_{14} \mathrm{H}_{18} \mathrm{~N}_{4} \mathrm{O}_{4} \mathrm{~S}_{2} \\
\cdot \mathrm{CH}_{2} \mathrm{Cl}_{2}\end{array}$ & $\begin{array}{l}\mathrm{C}_{14} \mathrm{H}_{18} \mathrm{~N}_{4} \mathrm{O}_{4} \mathrm{~S}_{2} \\
\cdot \mathrm{CHCl}_{3}\end{array}$ & $\begin{array}{l}\mathrm{C}_{14} \mathrm{H}_{18} \mathrm{~N}_{4} \mathrm{O}_{4} \mathrm{~S}_{2} \\
\text { - } \mathrm{C}_{4} \mathrm{H}_{8} \mathrm{O}_{2}\end{array}$ & $\begin{array}{l}\mathrm{C}_{14} \mathrm{H}_{18} \mathrm{~N}_{4} \mathrm{O}_{4} \mathrm{~S}_{2} \\
\text { - } \mathrm{C}_{4} \mathrm{H}_{8} \mathrm{O}_{2}\end{array}$ & $\begin{array}{l}\mathrm{C}_{14} \mathrm{H}_{18} \mathrm{~N}_{4} \mathrm{O}_{4} \mathrm{~S}_{2} \\
\text { - } \mathrm{C}_{5} \mathrm{H}_{5} \mathrm{~N}\end{array}$ & $\begin{array}{l}3\left(\mathrm{C}_{14} \mathrm{H}_{18} \mathrm{~N}_{4} \mathrm{O}_{4} \mathrm{~S}_{2}\right) \\
\cdot 2\left(\mathrm{C}_{7} \mathrm{H}_{8}\right)\end{array}$ \\
\hline Formula Mass & 428.52 & 455.37 & 489.81 & 458.55 & 458.55 & 449.54 & 1295.60 \\
\hline Crystal system & Monoclinic & Monoclinic & Monoclinic & Monoclinic & Triclinic & Triclinic & Tetragonal \\
\hline$a / \AA ̊$ & $16.0607(2)$ & 16.1404(3) & 15.5158(3) & 16.8293(3) & $9.2924(2)$ & $8.8104(9)$ & 11.7631(2) \\
\hline$b / \AA ̊$ & $17.4629(2)$ & 17.3754(3) & $18.0503(4)$ & $17.0275(3)$ & $11.7070(3)$ & 11.3513(13) & 11.7631(2) \\
\hline$c / \AA$ & $8.4560(1)$ & $8.2332(2)$ & $8.5922(2)$ & $8.2545(2)$ & $12.1305(3)$ & $12.500(2)$ & 48.5257(8) \\
\hline$\alpha /^{\circ}$ & 90.00 & 90.00 & 90.00 & 90.00 & $65.555(2)$ & $66.635(7)$ & 90.00 \\
\hline$\beta /{ }^{\circ}$ & 111.432(3) & 110.996(3) & 112.739(3) & 111.119(3) & $68.864(2)$ & 77.989(6) & 90.00 \\
\hline$\gamma /{ }^{\circ}$ & 90.00 & 90.00 & 90.00 & 90.00 & 76.329(3) & 77.883(5) & 90.00 \\
\hline $\mathrm{V} / \AA^{3}$ & 2207.63(5) & 2155.66(8) & 2219.34(8) & 2206.54(8) & 1114.61(5) & 1111.1(2) & $6714.5(2)$ \\
\hline Temperature/K & $173(2)$ & $173(2)$ & $173(2)$ & $173(2)$ & $173(2)$ & $173(2)$ & $173(2)$ \\
\hline Space group & $C 2 / c$ & $C 2 / C$ & $C 2 / c$ & $C 2 / C$ & $P-1$ & $P-1$ & $P 4(3) 22$ \\
\hline$Z$ & 4 & 4 & 4 & 4 & 2 & 2 & 4 \\
\hline Meas. reflns. & 2547 & 2757 & 2817 & 2410 & 5447 & 6213 & 11130 \\
\hline Indep. reflns. & 1878 & 1781 & 1907 & 1647 & 3818 & 4056 & 5685 \\
\hline$R_{\text {int }}$ & 0.0234 & 0.0605 & 0.0476 & 0.0361 & 0.0504 & 0.0366 & 0.0399 \\
\hline$R_{1}(I>2 \sigma(I))$ & 0.0359 & 0.1591 & 0.0755 & 0.0633 & 0.0444 & 0.0530 & 0.0434 \\
\hline$w R\left(F^{2}\right)(I>2 \sigma(I))$ & 0.0936 & 0.4149 & 0.1918 & 0.1799 & 0.1089 & 0.0985 & 0.0987 \\
\hline$R_{1}$ (all data) & 0.0393 & 0.1739 & 0.0893 & 0.0704 & 0.0575 & 0.0757 & 0.0631 \\
\hline$w R\left(F^{2}\right)$ (all data) & 0.0967 & 0.4220 & 0.2058 & 0.1870 & 0.1175 & 0.1096 & 0.1084 \\
\hline GOF on $F^{2}$ & 1.047 & 1.172 & 1.034 & 1.105 & 1.022 & 1.077 & 1.043 \\
\hline
\end{tabular}

${ }^{\mathrm{a}}$ Low data completeness (83\%) and new crystallizations produced TE-dioxane II. ${ }^{\mathrm{b}}$ Contains one TE in a general position and half a TE on a twofold axis.

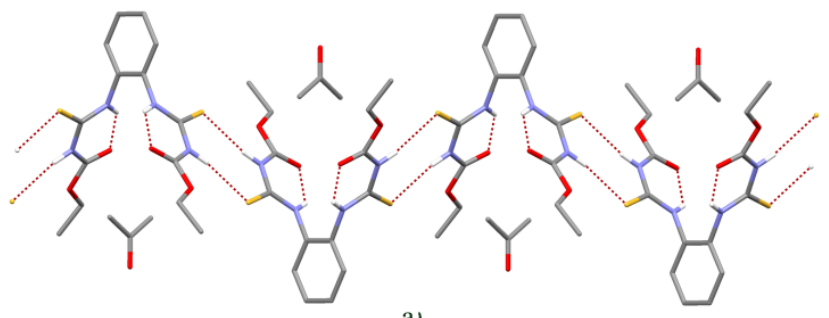

a)

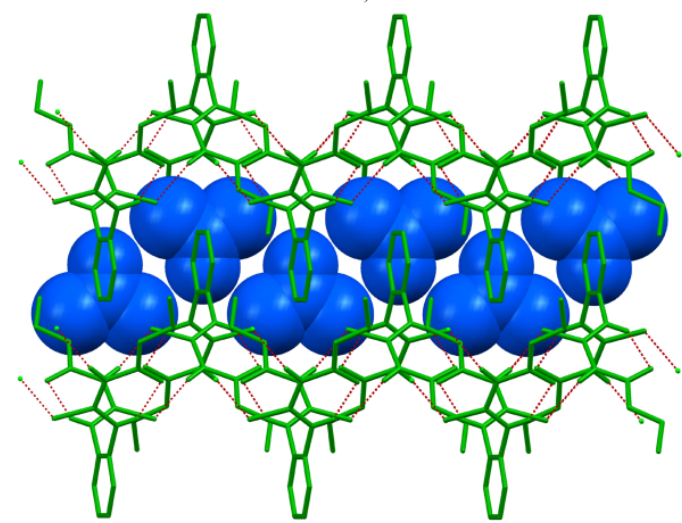

b)

Fig. 5 (a) Hydrogen bonded chains of TE acetone solvate and (b) the 5 channels of acetone in the structure with non-hydrogen bonding hydrogen atoms removed for clarity

According to the packing coefficients (Fig. 3) the hydrogen bonded pyridine and dioxane solvates pack more tighly than the isomorphous and toluene solvates, the only exception being 10 dioxane I, where the dioxane molecule more effectively fills the space available for it in the solvent channels. The isomorphous and toluene solvate crystals are very unstable when out of solution, desolvating in seconds/minutes, whereas the hydrogen bonded solvates, especially pyridine, are stable for hours.

${ }_{15}$ Comparison to TM solvates

Both TE and TM have a large amount of solvates (Table 4), though neither has been found to have a pure hydrate. Even though TM and TE are very similar it is difficult to find clearly similarly hydrogen bonded structures between them. The closest 20 analogues are the pyridine solvates, in which the pyridines are N$\mathrm{H} \cdots \mathrm{N}$ hydrogen bonded to the thiophanates.

Interestingly, no methanol and ethanol solvates of TE were found, whereas in the case of TM these solvates crystallize easily and are very stable. The methanol and ethanol solvates of TM are 25 isomorphous with each other and also very similar in hydrogen bonding to the acetone and cyclohexanone solvates. Likely, the formation of similar solvates of TE is hindered by packing problems regarding the larger size and more lipophilic nature of TE. The same kind of hydrogen bonding is, however, exhibited 30 by TE form III, which has voids in the structure and likely if the correct solvent and crystallization conditions could be found, a solvate with similar hydrogen bonding could crystallize.

TE, unlike TM, is able to make evident solvent channels that run in between the two arms of the molecule. In addition to 35 acetone, dioxane, dichloromethane and chloroform other solvent molecules of similar size (molecular volume of around 55 to 90 $\AA^{3}$, see Table 4) are suspected to fit in these channels and also form isomorphous solvates. Ethanol is perhaps too small, but propanol could be ideal and 1,2-dichloroethane and DMSO 40 possible solvate formers. The molecular volume of pyridine $(\sim 80$ $\AA^{3}$ ) is also such that it could fit in the solvent channels. The strong hydrogen bonding interactions between the solvent and the TE molecules, however, seem to determine the structure of the solvate in this case. Toluene is likely too large or unsuitably 45 shaped to fit in the solvent channels and thus makes another type of structure. 


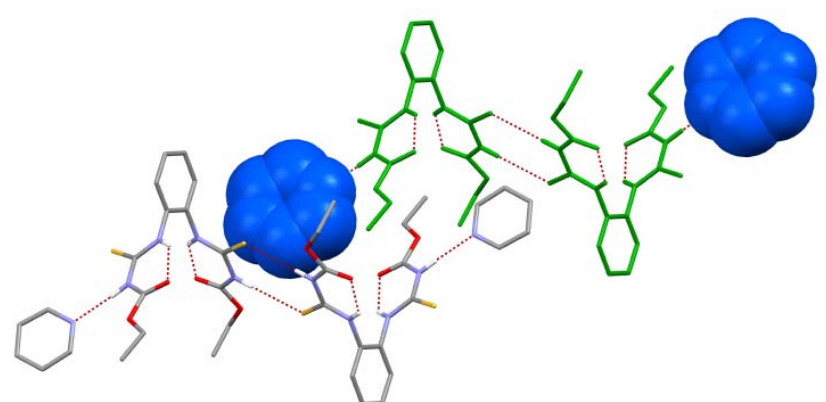

a)

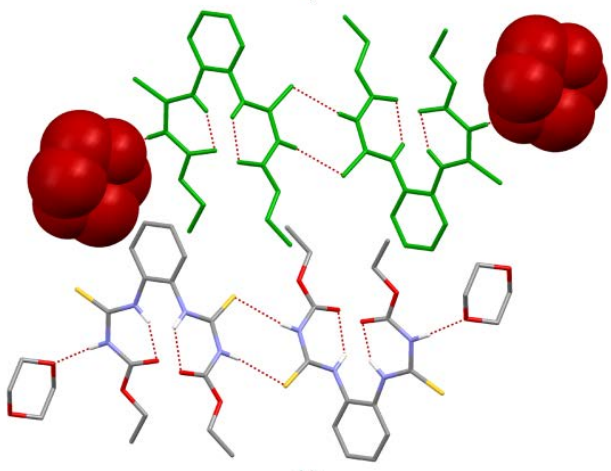

b)

Fig. 6 Two hydrogen bonded double pairs of (a) the TE pyridine solvate and (b) the TE dioxane solvate with non-hydrogen bonding hydrogen atoms removed for clarity

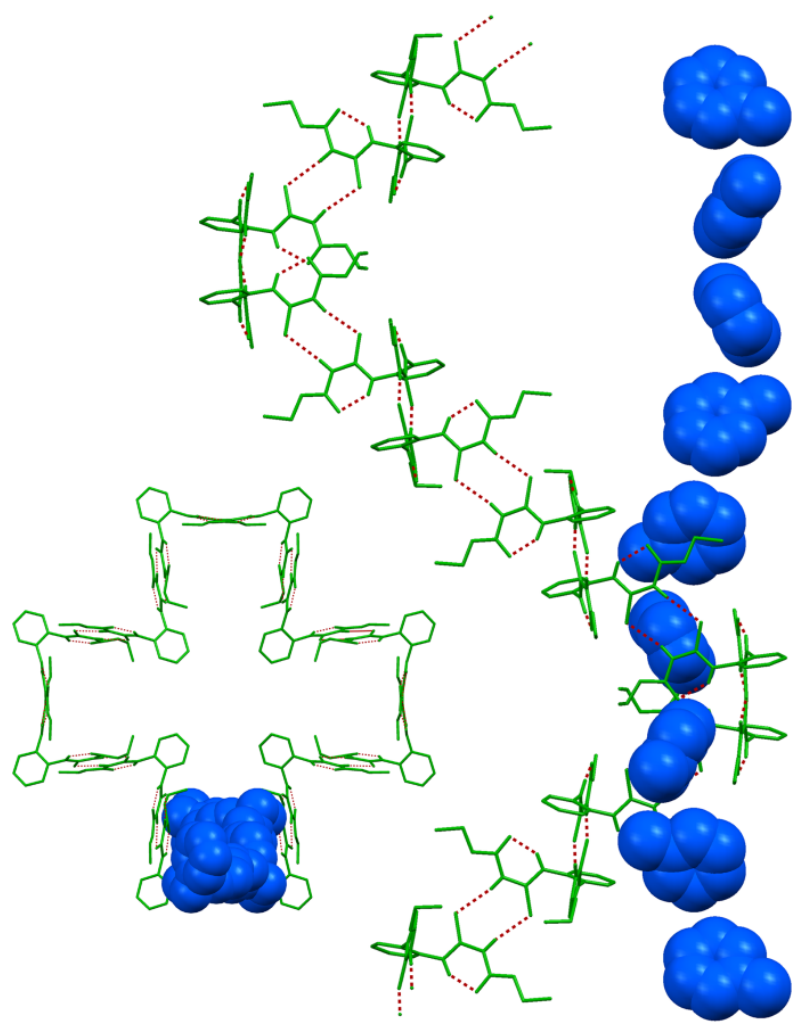

Fig. 7 One spiral in the toluene solvate from the side and top with toluene molecules in spacefill style and non-hydrogen bonding hydrogen atoms and disorder removed for clarity
Table 4 Molecular volumes ${ }^{a}$ of some common solvents and whether TM 10 or TE solvates have been found with these.

\begin{tabular}{cccc}
\hline Solvent & $\begin{array}{c}\text { Molecular } \\
\text { volume }\end{array}$ & TM solvate & TE solvate \\
\hline Water & 18.02 & $-{ }^{b}$ & - \\
Methanol & 37.21 & Yes & - \\
Acetonitrile & 46.06 & Yes $^{b}$ & - \\
Ethanol & 54.16 & Yes & - \\
DCM & 56.51 & Yes & Yes \\
Acetone & 64.74 & Yes & Yes \\
Chloroform & 70.07 & Yes & Yes \\
1/2-Propanol & $70.82 / 70.60$ & -- & -- \\
DMSO & 71.43 & Yes & - \\
1,2-Dichloroethane & 73.31 & Yes & - \\
THF & 77.99 & Yes & -- \\
Pyridine & 79.89 & Yes & Yes \\
Benzene & 87.04 & Yes & -- \\
Dioxane & 86.97 & Yes & Yes 2 \\
Dimethylacetamide & 94.09 & - & -- \\
Toluene & 100.61 & - & Yes \\
Cyclohexanone & 104.79 & Yes & -- \\
1,2-Dichlorobenzene & 111.12 & Yes & -
\end{tabular}

${ }^{a}$ Volumes from molinspiration.com. ${ }^{b}$ a $\mathrm{MeCN} / \mathrm{H}_{2} \mathrm{O}$ combination solvate - none found or -- not tested.

\section{Conclusions}

Four polymorphs and seven solvates of thiophanate-ethyl were 15 found and the crystal structures of all but polymorph IV solved. Thiophanate-ethyl and thiophanate-methyl are an example of when the applicability of a supramolecular synthon approach alone is limited. The behavior of $\mathrm{TE}$ is quite similar to thiophanate-methyl in that both willingly form solvates and 20 polymorphs, but with varying combinations of hydrogen bonding motifs and conformations of molecules. The $\mathrm{N}-\mathrm{H} \cdots \mathrm{O}=\mathrm{C}$ bond is the strongest possible hydrogen bond for TM and TE so one would expect it to show up more frequently than the weaker $\mathrm{N}$ $\mathrm{H} \cdot \mathrm{S}=\mathrm{C}$ hydrogen bond. This, however, is not the case, especially 25 for TE. Moreover, the solvate structures of the two actives vary greatly giving no general strategies for the design of cocrystals.

The challenge is that even though there are clearly a few best hydrogen bonding motifs, the conformational possibilities enable these to be used in a variety of combinations, which can not be 30 predicted. Another reason is the possibility for other weaker and not so directional, but combinatorially strong interactions like $\pi$ $\pi$-stacking and lipophilic effects. These can outweigh the propensity for certain kinds of hydrogen bonding synthons between molecules, like in the isomorphous solvates of TE. The 35 reduction of void space, is another factor since the energetic advantages in a certain kind of packing of molecules are difficult to predict.

We expect a supramolecular synthon approach to be most applicable with conformationally rigid molecules that have a 40 limited number of clear hydrogen bonding possibilities. Another case where a supramolecular approach could be used successfully, even with more complicated systems, would involve using reoccurring hydrogen bonds, like the hydrogen bond from TE/TM to the pyridine nitrogen. A further strategy for cocrystal 45 design with TE could be in fitting small cocrystallizing molecules in the channels of the isomorphous solvates. 


\section{Notes and references}

${ }^{a}$ University of Jyväskylä, Department of Chemistry, Nanoscience Center, P.O. Box 35, FIN-40014 University of Jyväskylä, Finland. Fax: +35814 2604756; Tel: +35814 2604242; E-mail: maija.nissinen@jyu.fi

$5{ }^{b}$ BASF SE, GVC/C - A030, 67056 Ludwigshafen, Germany. Fax: +49621 6020440; Tel:+49621 6049558, E-mail: heidi.saxell@basf.com

$\dagger$ Electronic Supplementary Information (ESI) available: [crystallographic data in CIF format]. See DOI: 10.1039/b000000x/

1 J. Bernstein, Polymorphism in Molecular Crystals, Oxford University 10 Press, United States, 2002; H. G. Brittain, Polymorphism in Pharmaceutical Solids, Marcel Dekker, Inc., New York, USA, 1999.

2 U. J. Griesser, in Polymorphism in the Pharmaceutical Industry, ed. R. Hilfiker, WILEY-VCH Verlag GmbH \& Co. KGaA, Weinheim, Germany, 2006, pp.211-234.

153 O. Almarsson and M. J. Zaworotko, Chem. Commun., 2004, (17), 1889-1896 (DOI:10.1039/b402150a); N. Blagden, M. de Matas, P. T. Gavan and P. York, Adv. Drug Deliv. Rev., 2007, 59, 617-630, (DOI:10.1016/j.addr.2007.05.011); P. Vishweshwar, J. A. McMahon, J. A. Bis and M. J. Zaworotko, J. Pharm. Sci., 2006, 95, 499-516 (DOI:10.1002/jps.20578).

4 G. R. Desiraju, Angew. Chem. Int. Ed. Engl., 1995, 34, 2311-2327; A. Nangia and G. R. Desiraju, Acta Crystallographica Section A, 1998, 54, 934-944 (DOI:10.1107/S0108767398008551); C. B. Aakeröy and D. J. Salmon, CrystEngComm, 2005, 7, 439-448

25 (DOI:10.1039/b505883j); G. R. Desiraju, Angew. Chem. Int. Ed., 2007, 46, 8342-8356 (DOI:10.1002/anie.200700534).

5 A. I. Kitaĭgorodskiň, Organic chemical crystallography, Consultants Bureau, New York, 1961.

6 M. A. Spackman and J. J. McKinnon, CrystEngComm, 2002, 4(66), 378-392.

7 Z.J. Li, W.H. Ojala and D.J.W. Grant, J. Pharm. Sci., 2001, 90, 152339.

8 M. C. Etter and J. C. MacDonald, Acta Cryst Sect.B, 1990, B46; 256262; J. Bernstein, R. E. Davis, L. Shimoni and N-L. Chung, Angew. Chem. Int. Ed. Engl., 1995, 34, 1555-1573.

9 E. Nauha, H. Saxell, M. Nissinen, E. Kolehmainen, A. Schäfer and R. Schlecker, CrystEngComm, 2009, 11, 2536-2547, (DOI:10.1039/b905511h).

10 Pat., WO/2008/096005, 08.02.2008.

4011 Eu Commission, OJEC, 23.11.2002, L 319, 3-11.

12 PANalytical B.V., 2006, 2.2b.

13 Z. Otwinowski, D. Borek, W. Majewski and W. Minor, Acta $\begin{array}{llll}\text { Crystallogr. } & \text { A., } & \text { 2003, } & \text { 59, }\end{array}$ (DOI:10.1107/S0108767303005488).

4514 G. M. Sheldrick, Acta Crystallogr. A., 2008, 64, 112-122, (DOI:10.1107/S0108767307043930).

15 C. F. Macrae, P. R. Edgington, P. McCabe, E. Pidcock, G. P. Shields, R. Taylor, M. Towler and J. van de Streek, J. Appl. Cryst., 2006, 39, 453-457, (DOI: 10.1107/S002188980600731X).

5016 Crystal packer program in Cerius ${ }^{2}$ program package, 4.6, Accelrys, 2001.

17 M.A. Neumann and M.-A. Perrin, J. Phys. Chem. B., 2005, 109, 15531-15541; M.A. Neumann, J. Phys. Chem. B., 2008, 112, 98109829.

5518 J. W. Steed, CrystEngComm, 2003, 5, 169-179; G. R. Desiraju, CrystEngComm, 2007, 9, 91-92 (DOI:10.1039/b614933b); K. M. Anderson and J. W. Steed, CrystEngComm, 2007, 9, 328-330.

19 The packing coefficient describes the tightness of the packing of molecules in a crystal, with a bigger value meaning tighter packing, and is calculated with the formula $\mathrm{C}(\mathrm{k})=\mathrm{Z} * \mathrm{~V}(\mathrm{~mol}) / \mathrm{V}$ (cell), where $\mathrm{Z}$ is the number of molecules in the unit cell, $\mathrm{V}(\mathrm{mol})$ is the molecular volume of the molecules and V(cell) the volume of the unit cell. For these calculations the disorder of the solvent was not taken into consideration.

6520 S. Brodersen, S Wilke, F. J. J.Leusen and G. Engel, Phys. Chem. Chem. Phys., 2003, 5, 4923-4931.

21 The $\mathrm{E}_{\mathrm{H}}$ is computed in Cerius ${ }^{2}$ by 12-10 potential with an angular term: $E_{H}=\left(C_{i j} / r_{i j}^{12}-D_{i j} / r_{i j}^{10}\right) \cos ^{4} \theta$, where $\theta$ is the angle between the donor, hydrogen and acceptor and $\mathrm{C}$ and $\mathrm{D}$ are empirical however, problematic for several reasons of which one is the power of 10 in the attractive term which makes the function sensitive for the separation of the hydrogen and acceptor atom as the energy drops rapidly with the increasing distance. The function is also sensitive for 75 the bonding angle due to the power of 4 in the cosine $\theta$ term. 